Instituto Internacional de Investigación y Desarrollo Tecnológico Educativo INDTEC, C.A.

DOI: https://doi.org/10.29394/scientific.issn.2542-2987.2017.2.5.14.262-282

OAI-PMH: http://www.indteca.com/ojs/index.php/Revista Scientific/oai

\title{
Expectativas y Percepciones del Servicio Educativo en la Universidad Nacional Abierta (Centro Local Mérida, Venezuela)
}

\author{
Autores: Teresa de Jesús Molina Gutiérrez \\ Universidad Nacional Abierta, UNA \\ teresaj.molina@gmail.com \\ Mérida, Venezuela \\ Belkis Molina de Sánchez \\ Universidad Valle del Momboy, UVM \\ belquismdavila@hotmail.com \\ Mérida, Venezuela \\ Luis Enrique González Zuñiga \\ Universidad de Los Andes, ULA \\ luisgonzalezprometeo@gmail.com \\ Mérida, Venezuela
}

\section{Resumen}

El objetivo de este estudio fue interpretar la relación existente entre las expectativas y las percepciones que posee el personal acerca de la calidad del servicio educativo que se ofrece en el Centro Local Mérida (Universidad Nacional Abierta), entonces, se empleó el modelo conceptual de calidad de servicio SERVQUAL, complementado con el enfoque cualitativo. Los informantes fueron 15 asesores, 20 estudiantes y 5 miembros del personal administrativo, los datos se recolectaron mediante la entrevista semiestructurada y se interpretaron con el análisis de contenido. Los resultados indican que no hay satisfacción con el servicio educativo o esa satisfacción es muy baja, porque las expectativas que se generan antes de recibirlo no son superadas por el valor que se percibe, lo cual es un indicador de ausencia de calidad de servicio. Por otra parte, lograr la calidad en el servicio prestado es responsabilidad de todos los involucrados ya que la complejidad de los roles cumplidos en la dialéctica educativa conlleva a que el personal actúe, simultáneamente, como clientes y como prestadores del servicio.

Palabras clave: servicio educativo; modelo SERQUAL; educación a distancia.

Fecha de Recepción: 06-03-2017

Fecha de Aceptación: 12-04-2017 


\title{
Expectations and Perceptions of the Educational Service at the Universidad Nacional Abierta (Local Center Merida, Venezuela)
}

\begin{abstract}
The objective of this study was to interpret the relationship between the expectations and the perceptions that the staff has about the quality of the educational service offered at the Mérida Local Center (Universidad Nacional Abierta), then the conceptual model of quality of service SERVQUAL, complemented with the qualitative approach. The informants were 15 advisors, 20 students and 5 administrative staff, the data were collected through the semi-structured interview and interpreted with the content analysis. The results indicate that there is no satisfaction with the educational service or that satisfaction is very low, because the expectations that are generated before receiving it are not surpassed by the perceived value, which is an indicator of the absence of quality of service. On the other hand, achieving quality in the service provided is the responsibility of all involved since the complexity of the roles fulfilled in the educational dialectic means that the staff act simultaneously as clients and as service providers.
\end{abstract}

Keywords: educational service; SERQUAL model; distance education.

Date Received: 06-03-2017

Date Acceptance: 12-04-2017 


\section{Introducción}

Es bien sabido que la universidad juega un rol estelar por la responsabilidad y el compromiso que contrae con la sociedad para ser agente de cambio y transformación, es un puente por donde se conduce el progreso al formar el talento humano que exige la sociedad y sus instituciones. Se establece así, una serie de relaciones e intercambios universidad-entorno, que es necesario gestionar con calidad y pertinencia para que el servicio, tanto en formación como en investigación y atención a la comunidad, sea óptimo y satisfaga las necesidades y exigencias en términos de conocimiento y progreso socio-cultural.

Al hablar de calidad en la educación superior se alude, entre otras cosas, a conceptos de relevancia, pertinencia y eficiencia. La relevancia, se articula con las necesidades sociales y las transformaciones exigidas, lo cual procura un tipo de educación adecuada para satisfacerlas. La pertinencia, se vincula con la coherencia de los objetivos, las situaciones de aprendizaje y las demandas sociales, haciendo énfasis en la adecuación de los niveles evolutivos y culturales de los sujetos educados. Y la eficiencia, es asociada a la racionalidad económica y administrativa, así como al óptimo empleo de los recursos disponibles, partiendo de una clara concepción de medios y fines.

En la búsqueda de una educación de calidad, el avance del conocimiento y la capacidad de cobertura alcanzada por la educación universitaria tradicional en un contexto determinado, permitió encontrar alternativas diferentes para atender las demandas existentes y garantizar inclusión a vastos sectores sociales. Surge así la educación a distancia, como modalidad innovadora, haciéndose cada vez más pertinente, viable y accesible a diferentes grupos etarios, que por diversos motivos se ven limitados 0 impedidos para seguir un proceso de formación presencial. Asimismo, el desarrollo masivo de múltiples herramientas tecnológicas facilita y agiliza la 
información y la comunicación en los estudios a distancia, lo que fortalece la modalidad.

Siguiendo a Cobo y Moravec (2011), el modelo universitario de educación a distancia está llamado a solventar muchas fallas, tanto en términos de cobertura e inclusión, como en pertinencia, eficiencia, flexibilidad e innovación. Sin embargo, estos autores afirman que los actuales modelos de educación están en crisis por los desfases entre las habilidades enseñadas y las requeridas en el mundo técnico-profesional, por la visión formativa de corto alcance que los define, lo que no permite atender adecuadamente los desafíos del futuro.

Por otra parte, las relaciones que se dan desde el ingreso del aspirante que desea cursar estudios en el Centro Local Mérida (UNA) con los orientadores, los asesores académicos y con las diferentes unidades operativas son fundamentales para medir la calidad del servicio educativo. Así pues, que las actitudes y valores que se difunden institucionalmente, la identidad con la modalidad a distancia, la oportunidad, pertinencia, rigurosidad y efectividad con que se atienda cada solicitud del que aprende, definen la calidad de los servicios académicos de apoyo y motivación.

En lo que respecta al servicio educativo prestado en el Centro Local Mérida es importante precisar que la matrícula estudiantil en el período comprendido entre los años 2009-2015, cayó en un 48,44\%; bien, por deserción o porque los estudiantes optaron por cambiar de Centro Local. Otro dato importante es que, en el período referido, solo el $40 \%$ del total de matriculados en las distintas carreras ofertadas, acudió a cumplir con los procesos de evaluación de los aprendizajes (presentación de pruebas y entrega de trabajos prácticos). En el mismo sentido, durante este período, (por lapso académico), ingresaron al Curso Introductorio no menos de 500 aspirantes, de los cuales el promedio de aprobados estuvo por encima del $50 \%$, y sólo se graduaron 100 estudiantes por lapso académico, estos datos 
dejan entrever el problema de deserción y el bajo rendimiento académico que presenta el Centro Local Mérida.

De cara a esta problemática, es necesario profundizar en la percepción presente en la comunidad académica, con respecto a la calidad de servicio y a las expectativas que llevan a estudiantes, asesores y personal administrativo a permanecer en la institución o a desertar. La calidad es un requisito esencial para las instituciones prestadoras de bienes y servicios, además de un derecho ciudadano.

Los servicios que ofrece la UNA a sus estudiantes, asesores, administrativos, comunidad, son intangibles y pertenecen a la clasificación de calidad subjetiva porque prevalece el juicio. Debido a que el interés de este estudio es abordar la calidad del servicio educativo prestado en el Centro Local Mérida, mediante la interpretación de las expectativas y de las percepciones de personal, para su medición se utilizó el modelo conceptual de calidad de servicio SERVQUAL; el mismo indaga a través de diagnósticos, las percepciones de los clientes con relación al servicio recibido. De acuerdo con Parasuraman, Zeithaml y Berry (1993), la calidad de servicio se puede determinar a través de las discrepancias entre las expectativas de sus demandantes y sus percepciones sobre la atención recibida.

\section{Objetivos de la investigación}

\subsection{Objetivo General}

Interpretar la relación existente entre las expectativas y las percepciones que poseen estudiantes, asesores y personal administrativo, sobre lo que deben ser los elementos tangibles, la fiabilidad, la capacidad de respuesta, la seguridad y la empatía institucional. (Centro Local Mérida, Universidad Nacional Abierta). 


\section{Referentes Teóricos}

En lo que al concepto de calidad de servicio se refiere, es preciso señalar que éste debe entenderse, según la UNESCO (1998), como la capacidad de proporcionar a los participantes el dominio de los códigos culturales básicos, la disposición para la participación democrática y ciudadana, el desarrollo de la capacidad y aptitud para resolver problemas y seguir aprendiendo, en función de los valores y actitudes que prevalecen en una sociedad, busca el bienestar y la calidad de vida para sus habitantes.

Considerar las necesidades, percepciones y expectativas del estudiante para medir calidad de servicio, es fundamental ya que éste como receptor del proceso formativo, debe ocupar el centro de la acción académica y los principios que definen el hecho docente. Para tal fin, hay que observar las particularidades que definen las percepciones y expectativas que manifiestan los estudiantes y los valores que nutren su comportamiento. De acuerdo con Lamas (2015) en el rendimiento académico del estudiante interactúan aspectos como las relaciones con los profesores, las aptitudes, la personalidad, los hábitos de estudio, las motivaciones y expectativas; la valoración del sistema de enseñanza, interacciones con el entorno al cual está asociado y con su nivel de satisfacción.

En opinión de Marcano (2016) es una necesidad lograr la transformación universitaria, para ello se requiere hacer converger lineamientos, principios y políticas que impulsen cambios para enrumbarse hacia una atención de calidad que beneficie a todos los involucrados.

La transformación de la universidad es una necesidad que también abarca la modalidad a distancia, evolución que encuentra un aliado perfecto en la gerencia universitaria, ésta, sostiene Sifuentes (2012), además de apoyarse en los elementos económicos financieros, debe recuperar el valor del sujeto para generar conocimientos desde todas sus acciones y cultivar el capital social. Generar la transformación involucra un mecanismo para abrirse 
e innovar, partiendo del principio que una formación integral de calidad debe incluir conocimientos, habilidades, valores y una actitud positiva hacia la vida, acordes a la realidad social del país, siempre favoreciendo el aprendizaje permanente y continúo vinculado con la problemática del contexto.

En lo que respecta al modelo SERVQUAL, (Parasúraman, Zeithaml y Berry, (ob.cit.) es una herramienta que define la calidad del servicio como la diferencia entre las percepciones reales por parte de los clientes y las expectativas que sobre él se habían formado previamente. De esta forma, un cliente/usuario valorará positiva o negativamente la calidad de un servicio en el que las percepciones que ha obtenido sean superiores o inferiores a las expectativas que tenía. Por ello, las compañías de servicios en las que uno de sus objetivos es la diferenciación mediante un servicio de calidad, deben prestar especial interés al hecho de superar las expectativas de sus clientes.

Otro aspecto que es esencial para ofrecer un servicio con calidad óptimo, oportuno y pertinente es el compromiso académico; este elemento, en el sistema de educación a distancia, requiere que las estrategias instruccionales y de apoyo a la instrucción se consoliden sobre una base de trabajo en equipo. Asimismo, Corredor (2012) considera que es determinante para esta modalidad tener en cuenta que los cambios sociales y científicos han incidido en una nueva manera de entender el conocimiento y el aprendizaje.

En este sentido, para la modalidad educativa a distancia es imprescindible la independencia del estudiante para fijar su ritmo de aprendizaje en función de sus responsabilidades y compromisos extra universitarios, porque facilita la función del docente como orientador y estimulador del proceso formativo. Así es esencial aportar los criterios de gestión que deben aplicarse para darle la atención pertinente y oportuna al que aprende, tanto en el plano académico como en el de apoyos administrativos auxiliares. 
En lo atinente a las expectativas y percepciones que determinan la calidad del servicio prestado, es necesario reconocer que un cliente es una persona u organización que demanda bienes o servicios proporcionados por el productor $o$ el proveedor de servicios para satisfacer una necesidad. Los clientes tienen necesidades y expectativas que debe considerar la organización y una necesidad es aquello que el consumidor o demandante realmente requiere; en tanto, una expectativa es una aspiración que puede ser conseguida o no con el servicio demandado. En el mismo sentido, es relevante tener en cuenta que las necesidades y las expectativas de las personas varían con el tiempo, ya sea por el crecimiento y desarrollo normal del individuo o por la influencia del entorno.

\section{Marco Metodológico}

\subsection{Tipo de Investigación}

En esta investigación el acercamiento indagatorio es interpretativo, de modo que se persiguió observar los fenómenos, comprender y percibir la vivencia (Martínez, 2009) acumulada sobre las expectativas y percepciones que tienen los usuarios acerca del servicio educativo que brinda el Centro Local Mérida, en interacción con el contexto, así como se enfatiza en el individuo y en su experiencia subjetiva.

Entonces, siguiendo a Parasuraman, Zeithaml y Berry (ob.cit.) se buscó comprender las dimensiones que conforman el constructo calidad de servicio a partir de la indagación sobre las expectativas y las percepciones que los usuarios tienen de la implementación de la educación a distancia en la institución seleccionada, lo cual se concretó al indagar sobre los siguientes aspectos: elementos tangibles, fiabilidad, capacidad de respuesta, seguridad y empatía institucional.

En cuanto a los informantes se escogieron de manera intencional, de modo que se integraron por: 15 asesores (contar con más de 5 años de 
servicio y haber ejercido cargos directivos), 20 estudiantes (haber aprobado el tercer semestre de la carrera), 5 miembros del personal administrativo (contar con más de 5 años de servicio, haber ejercido cargos de jefatura de unidades).

Por su parte, la técnica de recolección de la información fue la entrevista y como instrumento se empleó la entrevista semiestructurada, estos datos se analizaron mediante la técnica de análisis de contenido. Las entrevistas (organizadas en 4 y 5 interrogantes abiertas) dirigidas a todos los informantes recogieron información de acuerdo con las dimensiones establecidas por el modelo SERVQUAL:

a. elementos tangibles (instalaciones, personal, equipos, materiales).

b. Fiabilidad (entrega del servicio oportuno y confiable).

c. Capacidad de respuesta (buena disposición, servicio expedito).

d. Seguridad (atención, cortesía, confiabilidad y seguridad).

e. Empatía (atención individualizada al cliente).

\subsection{Interpretación de la Información}

El modelo SERVQUAL mide las dimensiones de la calidad de los servicios mediante una escala numérica: en atención a que nuestra perspectiva es cualitativa hicimos un ajuste al respecto, por lo que se establecieron diferencias entre las expectativas y percepciones a partir de criterios descriptivos. Así se analizaron las expectativas y percepciones de cada grupo de informantes, (estudiantes, administrativos, asesores), para las cinco categorías consideradas: elementos tangibles, confiabilidad, capacidad de respuesta, seguridad y empatía. De manera que se obtuvo lo siguiente:

\subsubsection{Estudiantes}

Expectativas: En lo que respecta a las expectativas de los estudiantes que aluden al servicio esperado, predomina una tendencia a esperar un servicio educativo en el que los elementos tangibles, la confiabilidad, la 
capacidad de respuesta, la seguridad y la empatía como criterios que garantizan la calidad respondan a: infraestructura adecuada a las necesidades de la población estudiantil, que sea funcional, que se optimice el aprovechamiento de los espacios y que especialmente, satisfaga los requerimientos de las personas con diversidad funcional. También se espera contar con equipos actualizados, y en cantidad suficiente, materiales instruccionales digitalizados, pedagógicos, actualizados, atención oportuna, personalizada y cordial, suficiente personal, bien formado y que ofrezca un trato amable, aplicación de principios de la educación a distancia y del humanismo, así como una comunicación horizontal.

Percepciones: Entre las percepciones más comunes de este mismo grupo, referidas al servicio recibido y tomando en cuenta las categorías referidas previamente, se percibe un importante nivel de insatisfacción: equipos desactualizados, los horarios de atención no responden a las necesidades, escasez de textos, son complejos y pesados, poca flexibilidad, amabilidad ocasional, lentitud en las respuestas, poca aplicabilidad del modelo conceptual de la educación a distancia, rigidez en la evaluación, poca comunicación, escasa empatía.

Como se puede observar las diferencias que existen entre las expectativas (servicio esperado) y las percepciones (servicio recibido) que tienen los estudiantes sobre la calidad de servicio indican existen expectativas muy ambiciosas con relación a lo que previamente esperan que sea el servicio que el Centro Local Mérida les pueda entregar, las cuales surgen de sus necesidades y experiencias, de modo que solicitan: Que se pueda contar con video beam, televisor, cámaras de video y fotografías...

Lo que contrasta abiertamente con las percepciones que se han hecho y que le permiten estimar y valorar de qué manera la institución está cumpliendo con la entrega del servicio: "Yo esperaría que los actualizarán, pues las pantallas vibran mucho y la disponibilidad de los horarios de estos 
son muy cortos". "He observado algunos textos con información caduca, la calidad del material instruccional es muy frágil".

En consecuencia, la brecha que existe entre ambas categorías se integra por juicios que realiza el estudiante y que permiten medir la calidad, por lo que esas valoraciones evidencian que existen importantes debilidades en lo que a las instalaciones físicas, equipos, personal y materiales de comunicación, se refiere, en la ejecución del servicio, en la capacidad de respuesta, en los conocimientos y atención mostrados por el personal de la Institución, así como en la atención individualizada ofrecida a los estudiantes. Ello incide directamente en que no haya satisfacción o ésta sea muy baja, porque las expectativas que genera antes de recibir el servicio no son superadas por el valor que percibe una vez que lo ha recibido. Esa brecha indica que los estudiantes como clientes no están satisfechos, lo que representa un indicador de ausencia de calidad de servicio.

Un fundamento conceptual de la modalidad a distancia afirma que lo importante es el estudiante y sus necesidades, asimismo, Corredor (2012) llama la atención sobre la necesidad de atender al estudiante del modo más apropiado ya que ellos son la razón fundamental que justifica la existencia del sistema.

Constatar que no se está ofreciendo un servicio de calidad a los estudiantes es preocupante porque ello es determinante para obtener las metas institucionales y para estar en correspondencia con los planteamientos de la UNESCO (2016), quien establece como objetivo mundial lograr una educación de calidad que llegue a todos y les ofrezca posibilidades de aprendizaje continuas y permanentes. Es indiscutible que la institución tiene la responsabilidad y la obligación de esforzarse al máximo por facilitar todos los recursos que propicien que el estudiante adquiera confianza y capacidad para responder exitosamente al trabajo universitario, ello se logra incorporando en 
un todo coherente las unidades que integran el sistema cuyo eje debe ser satisfacer los requerimientos de los estudiantes.

Visión que adquiere mayor pertinencia si se considera que en la modalidad a distancia, especialmente, es necesario facilitar al estudiante la adquisición de una serie de fortalezas que le ayudarán a superar los obstáculos que se presentan en un ambiente de estudio, cuya naturaleza involucra retos complejos como la autoformación, eventual orientación del asesor, dedicación en tiempo parcial al estudio, formación como actividad secundaria, entre otros.

De modo que es contundente que deba existir reciprocidad en el aporte que hacen los involucrados en la entrega del servicio, ya que tanta responsabilidad tienen docentes y administrativos de atender adecuadamente a los estudiantes como éstos tienen el deber de participar activamente en su proceso de formación, logrando buen rendimiento, acudiendo a las convocatorias, siendo honesto en la evaluación, asumiéndolos compromisos, aspectos inherentes al desempeño estudiantil, exigencias que, en elevados porcentajes, no cumplen.

\subsubsection{Personal Administrativo}

Expectativas: Al analizar cuáles son las expectativas, los deseos o necesidades que tiene el personal administrativo en las categorías: elementos tangibles, confiabilidad, capacidad de respuesta, seguridad y empatía, sobre lo que consideran debería ser el servicio que les tendrían que ofrecer; las tendencias indican lo siguiente: equipos tecnológicos actualizados y suficientes, seguridad, sentido de pertenencia, compromiso, personal actualizado y suficiente, comunicación eficaz, solidaridad, proactividad, mejoras laborales.

Percepciones: De acuerdo con Parasuraman, Zeithaml, y Berry los aspectos más representativos acerca de "las creencias de los consumidores 
relativos al servicio recibido" (ob.cit.: 14) son: infraestructura no adecuada, procesos rutinarios y manuales, equipos obsoletos y limitados, poco mantenimiento, ruidos en la comunicación, poca motivación, personal comprometido y eficiente, empatía, poco trabajo en equipo, divisiones grupales y pocas reivindicaciones laborales.

Como se puede observar los aspectos más importantes de cada categoría definen la calidad del servicio, por lo tanto se puede concluir, una vez más, que existen diferencias importantes entre expectativas y percepciones, lo que amplía esa brecha significativa para mostrar que de acuerdo con la opinión de este personal hay ineficiencias en la gestión de los servicios, lo que se traduce en un servicio pobre y de mala calidad, esto se corrobora en las siguientes opiniones "La instancia administrativa en pocas ocasiones responde a las necesidades y requerimientos". "Competencia desleal, falta de tolerancia y comunicación, falta de compañerismo y falta de ayuda y orientación al personal que ingresa nuevo al centro local", "Muchos, incluyéndome, aprenden de manera empírica y con el tiempo la modalidad de educación a distancia y esto hace que no ayudemos de manera afectiva a los estudiantes".

La insatisfacción de estos clientes se relaciona directamente con que la institución no les ofrece el suficiente apoyo en sus procesos de formación, no les aporta los insumos para cumplir con sus funciones, no les acoge en un clima organizacional armónico, no les satisface sus demandas laborales y no los motiva. El que este personal perciba mala calidad en el servicio debe preocupar al proveedor ya que el modelo de educación a distancia no puede prescindir del apoyo de las instancias administrativas, ellas sostienen el servicio académico. Lo que corroboran Rubinsztejn y Palacios (2012) al puntualizar que el énfasis de la gestión debe centrarse en el servicio educativo para luego pasar a una gestión dual, que considere tanto el servicio educativo como el funcionamiento operativo. 


\subsubsection{Personal Docente (Asesores)}

Expectativas: En lo que respecta a las expectativas del personal docente (asesores) sobre el servicio esperado, en las categorías: elementos tangibles, confiabilidad, capacidad de respuesta, seguridad y empatía, predomina lo siguiente: Disponer de recursos tecnológicos, actualización en la formación profesional y en materiales instruccionales, identidad institucional, planta física funcional, proactividad, comunicación eficaz, planificación, eficiencia, trabajo en equipo, autonomía, evaluación adaptada al sistema educativo, respuestas eficientes y oportunas, calidad, proactividad, aplicación principios educación a distancia, retroalimentación, acompañamiento, reconocimiento y compromiso.

Percepciones: Las percepciones que se ha hecho este grupo una vez que ha vivenciado cómo se le entrega el servicio se pueden resumir en estos aspectos: Infraestructura poco funcional, escaso mantenimiento, obsolescencia de materiales instruccionales y equipos, disminuido compromiso, servicio regular, poca atención individualizada, divisiones grupales, conformismo, desmotivación, centralismo, escasa credibilidad en el sistema de evaluación, poca armonía, improvisación, centralización de decisiones, obstaculización de procesos, baja capacidad de respuesta, incumplimiento de funciones, limitada aplicación de estrategias andragógicas, predisposición, escasa comunicación efectiva y distorsión de la información, trato inapropiado y descortés, desfavorable clima organizacional, aislamiento, poco reconocimiento a valores académicos e injusticia.

Al comparar los criterios involucrados en las expectativas y percepciones, se puede puntualizar que existe una gran brecha entre ambas categorías, lo que se concreta en una inconformidad importante pues los docentes expresan expectativas que visualizan un servicio de excelente calidad, lo que se puede observar en afirmaciones como: "Se debería responder oportunamente a las necesidades que presentan los académicos 
para realizar su trabajo", "En el desempeño de mi rol como asesora considero que necesito ser proactiva para estimular el desempeño positivo de mis estudiantes, la proactividad y mi compromiso son mis principios dentro de esta institución",

Estas expectativas cargadas de ideales sobre lo que esperan los asesores sea el funcionamiento de los distintos procesos educativos, son superadas considerablemente por las percepciones que traducen su vivencia, expresan su subjetividad y además indican descontento sobre el servicio obtenido, pues cuestionan abiertamente la calidad al sostener que: "Existen vicios que vienen de años, hay que luchar para sanear... no sé hasta dónde eso es viable, escapa al centro local. Considero que pasa en todos los Centros Locales".

Con relación a los equipos, destaca la escasez de los mismos y el deterioro de los pocos disponibles para el cumplimiento de funciones docentes y administrativas, lo cual representa una gran debilidad para la utilización de la tecnología en un sistema de estudios a distancia y que obliga, en diversas ocasiones y en forma de paliativo, a trasladar equipos personales al Centro Local.

De igual manera percibo que no hay ninguna orientación hacia mí como asesor; además, los estudiantes de nuevo ingreso muestran muchas deficiencias básicas que los impacta negativamente y los decepciona; por ejemplo, pierden materias por no tener clara la importancia de leer el Plan de Curso; desconocen dónde se presentan las pruebas; no manejan aspectos básicos de la evaluación.

Desde luego que en esta relación existe un proveedor llamado UNA y un cliente personificado en el asesor, su postura como cliente le indica que la institución además de suministrarle lo necesario para cumplir exitosamente con su rol de mediador del aprendizaje, también tiene el deber de satisfacer sus expectativas en lo que respecta a mejoras laborales, crecimiento 
profesional, clima organizacional armónico, buena comunicación, recursos y materiales de aprendizaje actualizados.

Pretensiones que se justifican ampliamente pues los asesores deberían contar con los recursos para brindar una excelente atención que vaya en beneficio de su principal cliente, el estudiante. Dada la importancia de ese rol y en correspondencia con lo que se desprende de las percepciones, exigen al proveedor del servicio mejoras constantes a su formación profesional, así como otros valores agregados que le generen un clima laboral confortable y armónico.

Este grupo a la vez que se muestra como la cara visible del servicio educativo, también es un cliente insatisfecho. Sus percepciones evidencian una actitud muy crítica ante el tipo de respuesta que se le ofrece y un reclamo de respaldo de la institución ya que sus percepciones les corrobora que no ha habido garantía de satisfacción de sus necesidades. Esa insatisfacción repercute en la calidad de su desempeño, afectando a los demás elementos que integran el sistema y además limitando su desarrollo profesional.

\section{Conclusiones}

De acuerdo con las percepciones de los usuarios la calidad del servicio que ofrece el Centro Local Mérida, no responde a un estándar de calidad. Al observar la cantidad de elementos que intervienen cuando estudiantes, administrativos y asesores valoran la calidad del servicio, existe una dualidad en ello, pues cada uno se postula como evaluador de la institución a partir de lo que son sus expectativas y sus percepciones, exigiendo de alguna manera que las percepciones superen a las expectativas, lo que significa un servicio de calidad, sin embargo, en esa dialéctica se percibe también que a la vez cada uno se siente evaluado por cuanto su actuación en la entrega del servicio determina la calidad o no de la misma. Ello evidencia la multidimensionalidad de la evaluación de la calidad y la complejidad que reviste las actitudes de 
estudiantes, docentes y administrativos al emitir juicios de valoración que critican un servicio de mala calidad en el que a la vez son responsables de la prestación de un servicio con esa calificación.

El nivel de importancia de estos resultados debe determinar que la universidad realice un verdadero esfuerzo por conocer y comprender las necesidades de su comunidad universitaria, asimismo, se requiere abundancia de estudios sistemáticos para analizar y describir el modo en que estas necesidades inciden en la percepción del valor del servicio.

\section{Referencias}

Cobo R. y Moravec, J. (2011). Aprendizaje Invisible. Hacia una nueva ecología de la educación. Barcelona: Universidad de Barcelona.

Corredor, Z. (2012). Competencias deseables para la asesoría académica adistancia mediada por TIC. Trabajo de ascenso no publicado. Universidad Nacional Abierta.

Lamas, H. (2015). Sobre el rendimiento escolar. Propósitos y Representaciones, 3(1), 313-386. Recuperado de: https://dialnet.unirioja.es/descarga/articulo/5475216.pdf

Marcano, J. (2017). Análisis Crítico de la Gerencia y Transformación en la Educación Universitaria, desde Diversos Postulados. Revista Scientific, 275-294. Recuperado de:

https://doi.org/10.29394/scientific.issn.2542-2987.2017.0.0.15.275-294 Martínez, M. (2009). Ciencia y arte de la metodología cualitativa. Métodos hermenéuticos, métodos fenomenológicos, métodos etnográficos. México: Trillas.

Parasúraman, A.; Zeithaml, V. y Berry, L. (1993). Calidad Total en Gestión de Servicios: cómo lograr equilibrio entre las percepciones y las expectativas de los consumidores. Madrid: Díaz de Santos. 
Rubinsztejn, M. y Palacios; M. (2012). El efecto del tiempo en la percepción de la calidad del servicio educativo, en Revista Iberoamericana de Educación. Ediciones OEI. Nro. 54. Recuperado de: http://rieoei.org/rie54a09.htm

Sifuentes. A. (2012). Reflexión teórica sobre la gerencia universitaria desde un enfoque complejo e integrador del capital social y la responsabilidad social universitaria. Tesis doctoral no publicada, Universidad de Los Andes, Mérida.

UNESCO. (1998). Declaración Mundial sobre la Educación Superior en el Siglo XXI: Visión y Acción y Marco de Acción Prioritario para el Cambio y el Desarrollo de la Educación Superior. Recuperado de: http://www.unesco.org/education/educprog/wche/declaration spa.htm UNESCO. (2016). La educación al servicio de los pueblos y el planeta. Creación De servicios sostenibles para todos. Recuperado de: http://unesdoc.unesco.org/images/0024/002457/245745s.pdf 


\section{Teresa de Jesús Molina Gutiérrez}

Nacida en Venezuela. Magister en Lingüística y en Literatura Iberoamericana, especialista en Metodología de la Investigación, $\mathrm{PhD}$. en Lingüística, egresada de la Universidad de Los Andes. Profesora Titular de pregrado y postgrado en la Universidad Nacional Abierta (UNA) y en la Universidad Pedagógica Experimental Libertador (UPEL), integrante del Grupo de Investigación: NIEME, líneas de investigación: análisis del discurso, educación universitaria, investigación cualitativa. Premio CONABA, investigadora PEII, participante como ponente, conferencista y tallerista en eventos de investigación nacionales e internacionales. 


\section{Belkis Molina de Sánchez}

e-mail: belquismdavila@hotmail.com

Nacida en Venezuela. Licenciada en Educación Integral, metodóloga, especialista en Planificación y Evaluación de la Educación, egresada de la Universidad Nacional Experimental Simón Rodríguez, candidata a doctora en Gerencia, de la Caribbean Internacional University-Curazao. Profesora en metodología de la investigación en la Universidad Valle del Momboy. Línea de investigación: educación, lectura y escritura, procesos culturales. Ponente y tallerista en eventos de intercambio científico. 


\section{Luis Enrique González Zuñiga}

e-mail: luisgonzalezprometeo@gmail.com

Nacido en Venezuela. Economista graduado en la

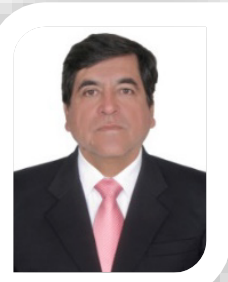
Universidad de los Andes (ULA), Especialización en Gerencia de Proyectos Multilaterales del IESA, Maestría en Ciencias Políticas en la Universidad de los Andes, PhD. en Patología Existencial e Intervención en Crisis de La Universidad Autónoma de Madrid (UAM). Prometeo SENESCYT, Ecuador. Profesor de pregrado y postgrado en varias Universidades: Ecuador: Universidad Laica "Eloy Alfaro" de Manabí (ULEAM), Universidad Nacional de Chimborazo (UNACH), Universidad de Guayaquil; España: Universidad Autónoma de Madrid; Venezuela: Universidad de los Andes de Venezuela, Universidad Nacional Experimental Sur del Lago (UNESUR), Universidad Nacional Experimental Simón Rodríguez.

El contenido de este manuscrito se difunde bajo una Licencia de Creative Commons ReconocimientoNoComercial-Compartirlgual 4.0 Internacional 